\title{
Pure Molecular Beam of Water Dimer
}

\author{
Helen Bieker, ${ }^{1,2,3}$ Jolijn Onvlee, ${ }^{1}$ Melby Johny, ${ }^{1,2}$ Lanhai He, ${ }^{1,} \|$ Thomas Kierspel, ${ }^{1,2,3, \S}$ \\ Sebastian Trippel, ${ }^{1,3}$ Daniel A. Horke, ${ }^{1,3, \ddagger}$ and Jochen Küpper ${ }^{1,2,3, *}$ \\ ${ }^{1}$ Center for Free-Electron Laser Science, Deutsches Elektronen-Synchrotron DESY, Notkestraße 85, 22607 Hamburg, Germany \\ ${ }^{2}$ Department of Physics, Universität Hamburg, Luruper Chaussee 149, 22761 Hamburg, Germany \\ ${ }^{3}$ Center for Ultrafast Imaging, Universität Hamburg, Luruper Chaussee 149, 22761 Hamburg, Germany
}

Spatial separation of water dimers from water monomers and larger water-clusters through the electric deflector is presented. A beam of water dimers with $93 \%$ purity and a rotational temperature of $1.5 \mathrm{~K}$ was obtained. Following strong-field ionization using a 35 fs laser pulse with a wavelength centered around $800 \mathrm{~nm}$ and a peak intensity of $10^{14} \mathrm{~W} / \mathrm{cm}^{2}$ we observed proton transfer and $46 \%$ of ionized water dimers broke apart into hydronium ions $\mathrm{H}_{3} \mathrm{O}^{+}$and neutral $\mathrm{OH}$.

\section{INTRODUCTION}

Hydrogen bonding between water molecules plays an important role in aqueous systems, e.g., for biomolecules that are surrounded by solvents. It is responsible for the unique properties of water, such as its high boiling point [1]. While hydrogen bonds have been studied extensively in many different molecular systems [2-8], one of the most important models remains the water dimer, somehow the smallest drop of water. Numerous studies have been conducted on this benchmark system and its structure with a single hydrogen bond is well known [912].

Water molecules and water-clusters have been studied using various techniques to describe dynamics such as proton motion [13] or chemical processes, e.g., reactive collisions [14]. For investigations of ultrafast molecular dynamics, such as energy and charge transfer across hydrogen bonds in molecular systems, photoion-photoion coincidence measurements at free-electron lasers are developing as a powerful tool $[8,15,16]$ and this approach was also used to study hydrogen bonding in the water dimer at a synchrotron [17]. Other spectroscopic techniques utilizing synchrotron facilities $[18,19]$ or table-top lasersystems $[5,6,20,21]$ further improved the knowledge about hydrogen bonding in water and water-clusters.

Most of these experiments investigating the dynamics of hydrogen-bonded systems would benefit from samples of identical molecules in a well-defined initial state. The widely used supersonic expansion technique provides cold molecular beams down to rotational temperatures of $<1 \mathrm{~K}$ [22-24]. However, cluster expansions do not produce single-species beams, but a mixture of various cluster stoichiometries. Hence, only low concentrations of specific species can be achieved. In the case of water molecules, supersonic expansion produces a cold beam of various water clusters [2] with a water dimer concentration of only a few percent $[17,25]$. This leads to small experimental event rates and requires long measurement times, e.g., in coincidence detection schemes [16, 17]. These experiments with a mixture of molecules in a molecular beam are only feasible if it can be disentangled which molecule was actually measured. Therefore, these mixtures severely limit the applicable techniques. A pure beam of water dimers would significantly speed up the measurements, when unwanted backgrounds from carrier gas and larger water-clusters are avoided, or simply enable such experiments.

The electrostatic deflector is an established method to spatially separate the molecules of interest from the carrier gas and to separate different species within a cold molecular beam [26]. This includes the separation of molecular conformers [27-30], individual quantum states of small molecules [31, 32], as well as specific molecular clusters $[24,33,34]$. The deflector was previously utilized in investigations of water, e. g., to determine the rotational temperatures of "warm" molecular beams of water [35], to separate its para and ortho species [32], and to measure the dipole moment of small water-clusters [36]. Alternatively, separation by the cluster species' distinct collision cross sections, i. e., by the transverse momentum changes due to scattering with a perpendicular rare-gas beam, was demonstrated [37]; this method is especially amenable to larger cluster sizes [38]. Such spatially separated singlespecies samples enable, for instance, advanced imaging applications of water-clusters using non-species-specific techniques, as well as the study of size-specific effects and the transition from single-molecule to bulk behavior.

\section{EXPERIMENTAL METHODS}

Here, the electrostatic deflector was used to spatially separate water dimers from water monomers as well as larger water-clusters in a molecular beam formed by supersonic expansion. The experimental setup was described previously [26, 39]. Briefly, liquid water was placed in the reservoir of an Even-Lavie valve [23], heated to $55^{\circ} \mathrm{C}$, seeded in 100 bar of helium, and expanded into vacuum with a nominal driving-pulse duration of $19.5 \mu$ s and at a repetition rate of $250 \mathrm{~Hz}$. The produced molecular beam was doubly skimmed, $6.5 \mathrm{~cm}(\varnothing=3 \mathrm{~mm})$ and $30.2 \mathrm{~cm}$ $(\varnothing=1.5 \mathrm{~mm})$ downstream from the nozzle, directed through the electrostatic deflector [40] of $154 \mathrm{~mm}$ length 


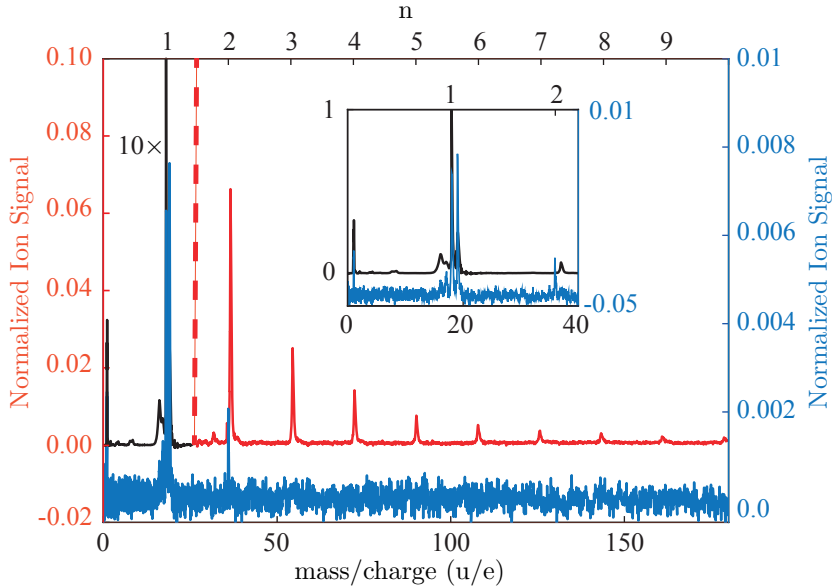

FIG. 1. TOF-MS in the center of the molecular beam as depicted in Fig. 2 with a deflector voltage of $0 \mathrm{kV}$ (black, red) and at a position of $+3 \mathrm{~mm}$ with a deflector voltage of $8 \mathrm{kV}$ (blue). For mass/charge $(m / q)$ ratios of $0 \ldots 30 \mathrm{u} / \mathrm{e}$, to the left of the dashed red line, the TOF-MS has been scaled by 0.1 . The inset shows the region of $m / q=0 \ldots 40 \mathrm{u} / \mathrm{e}$ enlarged; see text for details.

and with a nominal field strength of $50 \mathrm{kV} / \mathrm{cm}$ with an applied voltage of $8 \mathrm{kV}$ across the deflector, before passing through a third skimmer $(\varnothing=1.5 \mathrm{~mm})$. The deflector was placed $4.4 \mathrm{~cm}$ behind the tip of the second skimmer. In the center of a time-of-flight (TOF) mass spectrometer, $134.5 \mathrm{~cm}$ downstream from the nozzle, molecules were strong-field ionized by a 35 fs short laser pulse with a central wavelength around $800 \mathrm{~nm}$ and a pulse energy of $170 \mu \mathrm{J}$. Focusing to $65 \mu \mathrm{m}$ yielded a peak intensity of $\sim 10^{14} \mathrm{~W} / \mathrm{cm}^{2}$. The generated ions were accelerated toward a microchannel-plate detector combined with a phosphor screen and the generated signal was recorded with a digitizer. The valve, skimmers, and deflector were placed on motorized translation stages, which allowed movement of the molecular beam through the ionization laser focus and the recording of vertical molecular-beamdensity profiles without moving the laser focus, resulting in fixed imaging conditions [41-43].

While the employed strong-field ionization is a general, non-species specific ionization technique, it can also lead to fragmentation of molecules, such that recorded mass spectra (MS) do not directly reflect the composition of the molecular beam. In combination with the species-specific deflection process, however, this can be disentangled and, thus, even allows for the investigation of strong-fieldinduced fragmentation processes of a single species.

\section{RESULTS AND DISCUSSION}

TOF-MS of the direct and the deflected beams are shown in Fig. 1. The spectrum of the undeflected beam

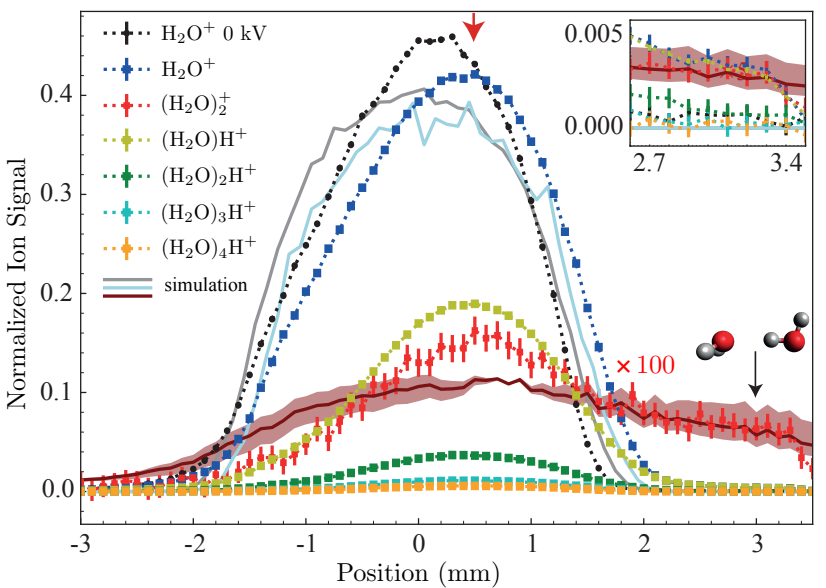

FIG. 2. Normalized measured vertical molecular-beam-density profiles (dashed lines) of water cation $\left(\mathrm{H}_{2} \mathrm{O}\right)^{+}$(blue), waterdimer cation $\left(\mathrm{H}_{2} \mathrm{O}\right)_{2}^{+}$(red), and protonated water-cluster ions $\left(\mathrm{H}_{2} \mathrm{O}\right)_{n} \mathrm{H}^{+}$up to $n=4$ (yellow, green, cyan, orange) with deflector voltages of $0 \mathrm{kV}$ (black circles) and $8 \mathrm{kV}$ (squares). Simulated vertical molecular-beam-density profiles of the undeflected water monomer (grey) as well as of the deflected water monomer (light blue) and water dimer (dark red) with a deflector voltage of $8 \mathrm{kV}$ are shown as solid lines. The shaded (dark red) area depicts the error estimate of the water dimer simulation due to the temperature uncertainty, $T_{\text {rot }}=1.5(5) \mathrm{K}$; see text for details. The black arrow indicates the position in the deflected beam where the TOF-MS shown in Fig. 1 was measured. The inset shows the deflection region enlarged with a magnification factor of 5 applied to the $\left(\mathrm{H}_{2} \mathrm{O}\right)_{2}^{+}$and $\left(\mathrm{H}_{2} \mathrm{O}\right)_{n} \mathrm{H}^{+}$signals.

shows water-cluster ions $\left(\mathrm{H}_{2} \mathrm{O}\right)_{n}^{+}$up to $n=2$ and protonated water-cluster ions $\left(\mathrm{H}_{2} \mathrm{O}\right)_{n} \mathrm{H}^{+}$up to $n=10$. Even larger clusters were likely formed in the supersonic expansion, but were not observed in the recorded TOF interval. We point out that all clusters that reach the interaction region are neutral clusters of the type $\left(\mathrm{H}_{2} \mathrm{O}\right)_{n}$, and protonated clusters must result from the interactions with the femtosecond laser, i. e., due to fragmentation during or after the strong-field-ionization process.

Vertical molecular-beam-density profiles for water ions $\left(\mathrm{H}_{2} \mathrm{O}\right)^{+}$, water dimer ions $\left(\mathrm{H}_{2} \mathrm{O}\right)_{2}^{+}$, and protonated watercluster ions $\left(\mathrm{H}_{2} \mathrm{O}\right)_{n} \mathrm{H}^{+}$up to $n=4$, with a potential difference of $8 \mathrm{kV}$ applied across the deflector, are shown in Fig. 2. For comparison, a field-free vertical profile for the water ion with $0 \mathrm{kV}$ across the deflector is also shown. The vertical molecular-beam-density profiles have been normalized to the area of the field-free spatial profile of the water ion. For visibility the water dimer profile has been scaled by a factor of 100 after normalization. While the field-free molecular beam profile is centered around $0 \mathrm{~mm}$, application of a voltage of $8 \mathrm{kV}$ to the deflector shifted the peak of water ions, water dimer ions, and protonated water-cluster ions by $+0.5 \mathrm{~mm}$, as indicated by the red arrow in Fig. 2. In addition, water dimer ions 
showed a broadening and an increase of signal at around $+3 \mathrm{~mm}$, indicated by a black arrow in Fig. 2 .

In the inset of Fig. 2 the region around $+3 \mathrm{~mm}$ is shown enlarged with a magnification factor of 5 applied to $\left(\mathrm{H}_{2} \mathrm{O}\right)_{2}^{+}$and $\left(\mathrm{H}_{2} \mathrm{O}\right)_{n} \mathrm{H}^{+}$with $n=1 \ldots 4$. The corresponding TOF-MS in the deflected part of the beam at a position of $+3 \mathrm{~mm}$ is highlighted in Fig. 1 by the blue line. Not just water dimer ions, but also hydronium ions, $\mathrm{H}_{3} \mathrm{O}^{+}$, and water ions, $\mathrm{H}_{2} \mathrm{O}^{+}$, showed an increased signal in the deflected beam. The shape of the vertical beam profiles for these ions matched the water dimer profile in the region of $2.8-3.5 \mathrm{~mm}$, indicating that they originated from the same parent molecule.

The water dimer ion was the largest non-protonated cluster measured in this setup. To verify that the water dimer ion was originating from the water dimer, the deflection behaviour of water-clusters inside the electrostatic deflector was simulated. Therefore, the Stark energies and effective dipole moments of water monomers and water-clusters were calculated with the freely available CMIsTARK software package [44] using rotational constants, dipole moments, and centrifugal distortion constants from the literature [11, 45-48], see Suppl. Inf. Table I; contributions of the polarizability to the Stark effect could safely be ignored $[40,49]$. The rotational constants of the water dimer are significantly smaller than for the water monomer, leading to a larger effective dipole moment for the water dimer than for water and a larger acceleration in the electric field in the deflector, see Fig. 3 and Table I of the Suppl. Inf. for further information.

The simulated vertical molecular-beam-density profiles of the water monomer and the water dimer are shown in Fig. 2. The deviations between the measured and simulated undeflected vertical beam profiles are ascribed to imperfect alignment of experimental setup, which was not taken into account in the simulations. Due to the rotational-state dependence of the Stark effect, the deflection of a molecular beam in an electrostatic field depends on the rotational temperature of the molecular ensemble [26] and the best fit for the profiles of the water monomer and the water dimer at a deflector voltage of $8 \mathrm{kV}$ was obtained assuming a Boltzmann population distribution of rotational states corresponding to $1.5(5) \mathrm{K}$.

Not only deflection of water-clusters measured as a mass of $36 \mathrm{amu}$, but also of water-clusters detected as protonated-clusters have been measured, for instance, for $\left(\mathrm{H}_{2} \mathrm{O}\right)_{2}^{+}$, as indicated by the red arrow and symbols in Fig. 2. Trajectory simulations for water-clusters $\left(\mathrm{H}_{2} \mathrm{O}\right)_{n}^{+}$with $n=3 \ldots 7$ using a rotational temperature of $T_{\text {rot }}=1.5(5) \mathrm{K}$ were performed to understand the origin of this deflection behavior. For the water hexamer three and for the water heptamer two conformers have been simulated, assuming an equal population of the conformers. These showed that, based on the different effective dipole moments, a different deflection is expected for different water-clusters, see Fig. 5 of the Suppl. Inf.. Since the detected protonated water-clusters arose from the strongfield fragmentation of larger neutral clusters in the interaction region, the measured vertical protonated-cluster density profiles are a superposition of several neutral water-cluster density profiles. Thus, it is not possible to compare the individual simulated molecular-beam-density profiles of neutral clusters directly with the measured protonated water-cluster density profiles. Instead, at each position of the deflection profile the signal from all waterclusters has been summed up, both for the simulated and the measured molecular-beam-density profiles. The result yields a comparable amount of deflection for simulated and measured molecular-beam-density profiles, see Suppl. Inf. Fig. 6. The shift of $0.5 \mathrm{~mm}$ can, therefore, originate from the superimposed molecular-beam-density profiles from different larger clusters due to fragmentation into smaller water-clusters. The same shift is visible for $\mathrm{H}_{2} \mathrm{O}^{+}$ and $\left(\mathrm{H}_{2} \mathrm{O}\right)_{2}^{+}$, which indicates that water-clusters are also fragmenting into $\mathrm{H}_{2} \mathrm{O}^{+}$and $\left(\mathrm{H}_{2} \mathrm{O}\right)_{2}^{+}$. Nevertheless, the simulation for water-clusters $n=1 \ldots 7$ shows that the water dimer deflected the most, reaching a position of $+3 \mathrm{~mm}$ and above, see Suppl. Inf. Fig. 4 and Fig. 5. Of all the other clusters considered, only the water hexamer in its prism and book forms reaches to a position up to $3.2 \mathrm{~mm}$ with the falling edge of the profile. In our experiments the water hexamer and higher order clusters have only been measured as fragments, such that the concentration and size distribution of neutral clusters in the molecular beam is unknown. However, the measured fragment distributions strongly suggest that significantly larger clusters are not present, since the ion signals decay exponentially and it is known that clusters primarily fragment through loss of single water molecules [50-52].

The TOF-MS in the deflected part of the beam, shown in Fig. 1, contains peaks corresponding to $\mathrm{H}^{+}, \mathrm{O}^{+}, \mathrm{OH}^{+}$, $\mathrm{H}_{2} \mathrm{O}^{+}$, and $\mathrm{H}_{3} \mathrm{O}^{+}$, in addition to the water dimer ion. As mentioned before the short-pulse ionization can lead to fragmentation. For the water dimer, two fragmentation channels were reported for electron-impact ionization with $70 \mathrm{eV}$ electrons [50]: either an $\mathrm{H}_{3} \mathrm{O}^{+}$ion and a neutral $\mathrm{OH}$ are formed or a $\mathrm{H}_{2} \mathrm{O}^{+}$ion and a neutral water monomer $\mathrm{H}_{2} \mathrm{O}$. Using a size-selection method and infrared spectroscopy, $\mathrm{H}_{2} \mathrm{O}^{+}$has been reported as a fragment of the water dimer [37]. Comparison of the vertical molecularbeam-density profiles of the deflected molecules allowed further investigation of the fragmentation channels of the water dimer. The measured vertical molecular-beamdensity profiles of these molecules showed a similar deflection behavior in the region of 2.8 to $3.5 \mathrm{~mm}$ as the water dimer, see Fig. 2 and Suppl. Inf. Fig. 1. The observed constant ratio of those fragments over this spatial region indicates that all these fragments originated from the water dimer.

Comparing the intensity of the fragments of the water dimer, $\mathrm{H}_{2} \mathrm{O}^{+}$and $\mathrm{H}_{3} \mathrm{O}^{+}$and $\left(\mathrm{H}_{2} \mathrm{O}\right)_{2}^{+}$, in the deflected beam, the fragmentation ratios of the intact water dimer 
were estimated. These showed that $46(7) \%$ of the water dimer fragmented into one ionized water molecule, while 46(4) $\%$ of the water dimer underwent most likely proton transfer and formed a hydronium ion. Only 8(2) \% of the water dimer present in the molecular beam stayed intact after ionization.

The actual number of water dimer molecules per shot in the deflected molecular beam was estimated to $\sim 0.8$ within the laser focus using the known fragmentation ratios of $\mathrm{H}_{2} \mathrm{O}^{+}$and $\mathrm{H}_{3} \mathrm{O}^{+}$, while the fragmentation channels of $\mathrm{H}^{+}, \mathrm{O}^{+}, \mathrm{OH}^{+}$have not been included. Taking the known fragmentation channels into account, the fraction of the water dimer within the molecular beam was evaluated. Comparing the ratios between the water dimer and all other species visible in the TOF, a water dimer fraction of $3.9(6) \%$ in the center of the undeflected beam and of $93(15) \%$ in the deflected beam, at a position of $+3 \mathrm{~mm}$, was achieved. Thus, using the electrostatic deflector the fraction of the water dimer within the interaction region could be increased by nearly a factor of 24 .

\section{CONCLUSIONS}

In summary, a high-purity beam of water dimers was created using the electrostatic deflector, which spatially separated water dimers from other species present in the molecular beam. The resulting water dimer sample had a purity of $93(15) \%$. The fragmentation products and ratios of the water dimer following strong-field ionization using a 35 fs laser pulse with a wavelength centered around $800 \mathrm{~nm}$ and peak intensity of $\sim 10^{14} \mathrm{~W} / \mathrm{cm}^{2}$ were studied, with 46(4) \% of the water dimer found to form a hydronium ion and 46(7) \% fragmenting into one water cation and one neutral water monomer, while $8(2) \%$ of the water dimer stayed intact. The deflection profiles could be simulated using a rigid-rotor model and an initial rotational temperature of $1.5(5) \mathrm{K}$.

The produced clean samples of water dimers are well suited for non-species-specific experiments, e.g., reactive-collisions, diffractive imaging, or ultrafast spectroscopies [14, 41, 53]. Even for experiments that can distinguish different species, for example photoion-photoion coincidence measurements [8, 54], the produced clean beams will enable significantly faster measurements of this important hydrogen-bonded model system, e. g., because unwanted backgrounds are avoided. Furthermore, the electrostatic separation technique can be used to separate different conformers [26], which could be highly interesting in the purification and studies of larger waterclusters that exhibit multiple conformers [55].

\section{ACKNOWLEDGMENTS}

This work has been supported by the Clusters of Excellence "Center for Ultrafast Imaging" (CUI, EXC 1074, ID 194651731) and "Advanced Imaging of Matter" (AIM, EXC 2056, ID 390715994) of the Deutsche Forschungsgemeinschaft (DFG), by the European Union's Horizon 2020 research and innovation program under the Marie Skłodowska-Curie Grant Agreement 641789 "Molecular Electron Dynamics investigated by Intense Fields and Attosecond Pulses" (MEDEA), by the European Research Council under the European Union's Seventh Framework Program (FP7/2007-2013) through the Consolidator Grant COMOTION (ERC-Küpper-614507), and by the Helmholtz Gemeinschaft through the "Impuls- und Vernetzungsfond". L.H. acknowledges a fellowship within the framework of the Helmholtz-OCPC postdoctoral exchange program and J.O. gratefully acknowledges a fellowship by the Alexander von Humboldt Foundation.

\section{SUPPORTING INFORMATION DESCRIPTION}

Supporting Information Available: Description of the fragmentation correction method and the trajectory simulations

॥ Permanent address: Institute of Atomic and Molecular Physics, Jilin University, Changchun 130012, China

$\S$ Present address: Department of Chemistry, University of Basel, Klingelbergstrasse 80, 4056 Basel, Switzerland

$\ddagger$ Present address: Institute for Molecules and Materials, Radboud University, Heijendaalseweg 135, 6525 AJ Nijmegen, The Netherlands

* Corresponding author:_jochen.kuepper@cfel.de; https://www.controlled-molecule-imaging.org

[1] G. A. Jeffrey, An Introduction to Hydrogen Bonding (Oxford University Press, 1997).

[2] K. Liu, J.D. Cruzan, and R.J. Saykally, "Water clusters," Science 271, 929-933 (1996).

[3] K. Nauta and R. E. Miller, "Formation of cyclic water hexamer in liquid helium: The smallest piece of ice," Science 287, 293 (2000).

[4] G. T. Dunning, D. R. Glowacki, T. J. Preston, S. J. Greaves, G. M. Greetham, I. P. Clark, M. Towrie, J. N. Harvey, and A. J. Orr-Ewing, "Vibrational relaxation and microsolvation of DF after F-atom reactions in polar solvents," Science 347, 530-533 (2015).

[5] G. Berden, W. L. Meerts, M. Schmitt, and K. Kleinermanns, "High resolution UV spectroscopy of phenol and the hydrogen bonded phenol-water cluster," J. Chem. Phys. 104, 972 (1996).

[6] Timothy M. Korter, David W. Pratt, and Jochen Küpper, "Indole- $\mathrm{H}_{2} \mathrm{O}$ in the gas phase. Structures, barriers to internal motion, and $\mathrm{S}_{1} \leftarrow \mathrm{S}_{0}$ transition moment orientation. Solvent reorganization in the electronically excited state," J. Phys. Chem. A 102, 7211-7216 (1998). 
[7] A. L. Sobolewski and W. Domcke, "Photoinduced electron and proton transfer in phenol and its clusters with water and ammonia," J. Phys. Chem. A 105, 9275-9283 (2001).

[8] X. Ren, E. Wang, A. D. Skitnevskaya, A. B. Trofimov, G. Kirill, and A. Dorn, "Experimental evidence for ultrafast intermolecular relaxation processes in hydrated biomolecules," Nat. Phys. 79, 1745 (2018).

[9] J. A. Odutola and T. R. Dyke, "Partially deuterated water dimers: Microwave spectra and structure," J. Chem. Phys. 72, 5062-5070 (1980).

[10] H. Yu and W. F. van Gunsteren, "Charge-on-spring polarizable water models revisited: From water clusters to liquid water to ice," J. Chem. Phys. 121, 9549-9564 (2004).

[11] T. R. Dyke, K. M. Mack, and J. S. Muenter, "The structure of water dimer from molecular beam electric resonance spectroscopy," J. Chem. Phys. 66, 498-510 (1977).

[12] T. R. Dyke and J. S. Muenter, "Molecular-beam electric deflection studies of water polymers," J. Chem. Phys. 57, 5011 (1972)

[13] T. Marchenko, L. Inhester, G. Goldsztejn, O. Travnikova, L. Journel, R. Guillemin, I. Ismail, D. Koulentianos, D. Céolin, R. Püttner, M. N. Piancastelli, and M. Simon, "Ultrafast nuclear dynamics in the doubly-core-ionized water molecule observed via auger spectroscopy," Phys. Rev. A 98, 063403 (2018).

[14] Ardita Kilaj, Hong Gao, Daniel Rösch, Uxia Rivero, Jochen Küpper, and Stefan Willitsch, "Observation of different reactivities of para- and ortho-water towards trapped diazenylium ions," Nat. Commun. 9, 2096 (2018).

[15] Rebecca Boll, Benjamin Erk, Ryan Coffee, Sebastian Trippel, Thomas Kierspel, Cédric Bomme, John D. Bozek, Mitchell Burkett, Sebastian Carron, Ken R. Ferguson, Lutz Foucar, Jochen Küpper, Tatiana Marchenko, Catalin Miron, Minna Patanen, Timur Osipov, Sebastian Schorb, Marc Simon, Michelle Swiggers, Simone Techert, Kiyoshi Ueda, Christoph Bostedt, Daniel Rolles, and Artem Rudenko, "Charge transfer in dissociating iodomethane and fluoromethane molecules ionized by intense femtosecond x-ray pulses," Struct. Dyn. 3, 043207 (2016).

[16] Thomas Kierspel, Imaging structure and dynamics using controlled molecules, Dissertation, Universität Hamburg, Hamburg, Germany (2016).

[17] T. Jahnke, H. Sann, T. Havermeier, K. Kreidi, C. Stuck, M. Meckel, M. Schöffler, N. Neumann, R. Wallauer, S. Voss, A. Czasch, O. Jagutzki, A. Malakzadeh, F. Afaneh, Th. Weber, H. Schmidt-Böcking, and R. Dörner, "Ultrafast energy transfer between water molecules," Nat. Phys. 6, 139-142 (2010).

[18] Bernd Winter, Emad F. Aziz, Uwe Hergenhahn, Manfred Faubel, and Ingolf V. Hertel, "Hydrogen bonds in liquid water studied by photoelectron spectroscopy," J. Chem. Phys. 126, 124504 (2007).

[19] Jared D. Smith, Christopher D. Cappa, Kevin R. Wilson, Benjamin M. Messer, Ronald C. Cohen, and Richard J. Saykally, "Energetics of hydrogen bond network rearrangements in liquid water," Science 306, 851-853 (2004).

[20] Frank N. Keutsch and Richard J. Saykally, "Inaugural article: Water clusters: Untangling the mysteries of the liquid, one molecule at a time," PNAS 98, 10533-10540 (2001).

[21] T. S. Zwier, "The spectroscopy of solvation in hydrogenbonded aromatic clusters," Annu. Rev. Phys. Chem. 47,
205-241 (1996).

[22] G. Scoles, ed., Atomic and molecular beam methods, Vol. 1 (Oxford University Press, New York, NY, USA, 1988).

[23] U. Even, J. Jortner, D. Noy, N. Lavie, and N. CossartMagos, "Cooling of large molecules below $1 \mathrm{~K}$ and He clusters formation," J. Chem. Phys. 112, 8068-8071 (2000).

[24] Melby Johny, Jolijn Onvlee, Thomas Kierspel, Helen Bieker, Sebastian Trippel, and Jochen Küpper, "Spatial separation of pyrrole and pyrrole-water clusters," Chem. Phys. Lett. 721, 149-152 (2019), arXiv:1901.05267 [physics].

[25] J. B. Paul, C. P. Collier, R. J. Saykally, J. J. Scherer, and A. O'Keefe, "Direct measurement of water cluster concentrations by infrared cavity ringdown laser absorption spectroscopy," J. Phys. Chem. A 101, 5211-5214 (1997).

[26] Yuan-Pin Chang, Daniel A. Horke, Sebastian Trippel, and Jochen Küpper, "Spatially-controlled complex molecules and their applications," Int. Rev. Phys. Chem. 34, 557590 (2015), arXiv:1505.05632 [physics].

[27] Frank Filsinger, Undine Erlekam, Gert von Helden, Jochen Küpper, and Gerard Meijer, "Selector for structural isomers of neutral molecules," Phys. Rev. Lett. 100, 133003 (2008), arXiv:0802.2795 [physics].

[28] Frank Filsinger, Jochen Küpper, Gerard Meijer, Jonas L. Hansen, Jochen Maurer, Jens H. Nielsen, Lotte Holmegaard, and Henrik Stapelfeldt, "Pure samples of individual conformers: the separation of stereo-isomers of complex molecules using electric fields," Angew. Chem. Int. Ed. 48, 6900-6902 (2009).

[29] Thomas Kierspel, Daniel A. Horke, Yuan-Pin Chang, and Jochen Küpper, "Spatially separated polar samples of the cis and trans conformers of 3-fluorophenol," Chem. Phys. Lett. 591, 130-132 (2014), arXiv:1312.4417 [physics].

[30] Nicole Teschmit, Daniel A. Horke, and Jochen Küpper, "Spatially separating the conformers of the dipeptide AcPhe-Cys- $\mathrm{NH}_{2}$," Angew. Chem. Int. Ed. 57, 13775-13779 (2018), arXiv:1805.12396 [physics]

[31] Jens H. Nielsen, Paw Simesen, Christer Z. Bisgaard, Henrik Stapelfeldt, Frank Filsinger, Bretislav Friedrich, Gerard Meijer, and Jochen Küpper, "Stark-selected beam of ground-state OCS molecules characterized by revivals of impulsive alignment," Phys. Chem. Chem. Phys. 13, 18971-18975 (2011), arXiv:1105.2413 [physics].

[32] Daniel A. Horke, Yuan-Pin Chang, Karol Długołęcki, and Jochen Küpper, "Separating para and ortho water," Angew. Chem. Int. Ed. 53, 11965-11968 (2014), arXiv:1407.2056 [physics].

[33] Sebastian Trippel, Yuan-Pin Chang, Stephan Stern, Terry Mullins, Lotte Holmegaard, and Jochen Küpper, "Spatial separation of state- and size-selected neutral clusters," Phys. Rev. A 86, 033202 (2012), arXiv:1208.4935 [physics].

[34] Hyun Sik You, Junggil Kim, Songhee Han, Doo-Sik Ahn, Jean Sun Lim, and Sang Kyu Kim, "Spatial isolation of conformational isomers of hydroquinone and its water cluster using the stark deflector," J. Phys. Chem. A $\mathbf{1 2 2}$, 1194 (2018).

[35] Ramiro Moro, Jaap Bulthuis, Jonathon Heinrich, and Vitaly V. Kresin, "Electrostatic deflection of the water molecule: A fundamental asymmetric rotor," Phys. Rev. A 75, 013415 (2007).

[36] Ramiro Moro, Roman Rabinovitch, Chunlei Xia, and Vitaly V. Kresin, "Electric dipole moments of water clusters from a beam deflection measurement," Phys. Rev. Lett. 
97, 123401 (2006).

[37] Udo Buck and Friedrich Huisken, "Infrared spectroscopy of size-selected water and methanol clusters," Chem. Rev. 100, 3863-3890 (2000).

[38] Christoph C. Pradzynski, Richard M. Forck, Thomas Zeuch, Petr Slavíček, and Udo Buck, "A fully size-resolved perspective on the crystallization of water clusters," Science 337, 1529-1532 (2012).

[39] Sebastian Trippel, Melby Johny, Thomas Kierspel, Jolijn Onvlee, Helen Bieker, Hong Ye, Terry Mullins, Lars Gumprecht, Karol Długołęcki, and Jochen Küpper, "Knife edge skimming for improved separation of molecular species by the deflector," Rev. Sci. Instrum. 89, 096110 (2018), arXiv:1802.04053 [physics].

[40] Jens S. Kienitz, Karol Długołęcki, Sebastian Trippel, and Jochen Küpper, "Improved spatial separation of neutral molecules," J. Chem. Phys. 147, 024304 (2017), arXiv:1704.08912 [physics].

[41] Jochen Küpper, Stephan Stern, Lotte Holmegaard, Frank Filsinger, Arnaud Rouzée, Artem Rudenko, Per Johnsson, Andrew V. Martin, Marcus Adolph, Andrew Aquila, Saša Bajt, Anton Barty, Christoph Bostedt, John Bozek, Carl Caleman, Ryan Coffee, Nicola Coppola, Tjark Delmas, Sascha Epp, Benjamin Erk, Lutz Foucar, Tais Gorkhover, Lars Gumprecht, Andreas Hartmann, Robert Hartmann, Günter Hauser, Peter Holl, Andre Hömke, Nils Kimmel, Faton Krasniqi, Kai-Uwe Kühnel, Jochen Maurer, Marc Messerschmidt, Robert Moshammer, Christian Reich, Benedikt Rudek, Robin Santra, Ilme Schlichting, Carlo Schmidt, Sebastian Schorb, Joachim Schulz, Heike Soltau, John C. H. Spence, Dmitri Starodub, Lothar Strüder, Jan Thøgersen, Marc J. J. Vrakking, Georg Weidenspointner, Thomas A. White, Cornelia Wunderer, Gerard Meijer, Joachim Ullrich, Henrik Stapelfeldt, Daniel Rolles, and Henry N. Chapman, "X-ray diffraction from isolated and strongly aligned gas-phase molecules with a free-electron laser," Phys. Rev. Lett. 112, 083002 (2014), arXiv:1307.4577 [physics].

[42] Stephan Stern, Lotte Holmegaard, Frank Filsinger, Arnaud Rouzée, Artem Rudenko, Per Johnsson, Andrew V. Martin, Anton Barty, Christoph Bostedt, John D. Bozek, Ryan N. Coffee, Sascha Epp, Benjamin Erk, Lutz Foucar, Robert Hartmann, Nils Kimmel, Kai-Uwe Kühnel, Jochen Maurer, Marc Messerschmidt, Benedikt Rudek, Dmitri G. Starodub, Jan Thøgersen, Georg Weidenspointner, Thomas A. White, Henrik Stapelfeldt, Daniel Rolles, Henry N. Chapman, and Jochen Küpper, "Toward atomic resolution diffractive imaging of isolated molecules with x-ray free-electron lasers," Faraday Disc. 171, 393 (2014), arXiv:1403.2553 [physics].
[43] Frank Filsinger, Jochen Küpper, Gerard Meijer, Lotte Holmegaard, Jens H. Nielsen, Iftach Nevo, Jonas L. Hansen, and Henrik Stapelfeldt, "Quantum-state selection, alignment, and orientation of large molecules using static electric and laser fields," J. Chem. Phys. 131, 064309 (2009), arXiv:0903.5413 [physics].

[44] Y.-P. Chang, F. Filsinger, B. Sartakov, and J. Küpper, "CMIstark: Python package for the stark-effect calculation and symmetry classification of linear, symmetric and asymmetric top wavefunctions in dc electric fields," Comp. Phys. Comm. 185, 339-349 (2014), arXiv:1308.4076 [physics].

[45] Frank C. DeLucia, Paul Helminger, and William H. Kirchhoff, "Microwave spectra of molecules of astrophysical interest V. Water vapor," J. Phys. Chem. Ref. Data 3, 211-219 (1974).

[46] L. H. Coudert and J. T. Hougen, "Analysis of the microwave and far infrared spectrum of the water dimer," Journal Of Molecular Spectroscopy 139, 259-277 (1990).

[47] Shelley L Shostak, William L Ebenstein, and John S Muenter, "The dipole moment of water. I. dipole moments and hyperfine properties of $\mathrm{H}_{2} \mathrm{O}$ and HDO in the ground and excited vibrational states," J. Chem. Phys. 94, 5875 (1991).

[48] N. P. Malomuzh, V. N. Makhlaichuk, and S. V. Khrapatyi, "Water dimer dipole moment," Russian Journal of Physical Chemistry A 88, 1431-1435 (2014).

[49] George Maroulis, "Static hyperpolarizability of the water dimer and the interaction hyperpolarizability of two water molecules," J. Chem. Phys. 113, 1813-1820 (2000).

[50] L. Angel and A. J. Stace, "Dissociation patterns of $\left(\mathrm{H}_{2} \mathrm{O}\right)_{n}^{+}$ cluster ions, for $\mathrm{n}=2-6$," Chem. Phys. Lett. 345, 277-281 (2001).

[51] Leonid Belau, Kevin R Wilson, Stephen R Leone, and Musahid Ahmed, "Vacuum ultraviolet (VUV) photoionization of small water clusters," J. Phys. Chem. A 111, 10075-10083 (2007).

[52] Xiaojie Liu, Wen-Cai Lu, C.Z. Wang, and K.M. Ho, "Energetic and fragmentation stability of water clusters (h2o)n, n=2-30," Chem. Phys. Lett. 508, $270-275$ (2011).

[53] Christopher J. Hensley, Jie Yang, and Martin Centurion, "Imaging of isolated molecules with ultrafast electron pulses," Phys. Rev. Lett. 109, 133202 (2012).

[54] Thomas Kierspel, Cédric Bomme, Michele Di Fraia, Joss Wiese, Denis Anielski, Sadia Bari, Rebecca Boll, Benjamin Erk, Jens S. Kienitz, Nele L. M. Müller, Daniel Rolles, Jens Viefhaus, Sebastian Trippel, and Jochen Küpper, "Photophysics of indole upon x-ray absorption," Phys. Chem. Chem. Phys. 20, 20205 (2018), arXiv:1802.02964 [physics].

[55] J. K. Gregory, D. C. Clary, K. Liu, M. G. Brown, and R. J. Saykally, "The water dipole moment in water clusters," Science 275, 814-817 (1997). 


\title{
Supplemental Material: Pure molecular beam of water dimer
}

\author{
Helen Bieker, ${ }^{1,2,3}$ Jolijn Onvlee, ${ }^{1}$ Melby Johny, ${ }^{1,2}$ Lanhai He, ${ }^{1,} \|$ Thomas Kierspel, ${ }^{1,2,3, \S}$ \\ Sebastian Trippel, ${ }^{1,2}$ Daniel A. Horke, ${ }^{1,2, \ddagger}$ and Jochen Küpper ${ }^{1,2,3, *}$ \\ ${ }^{1}$ Center for Free-Electron Laser Science, Deutsches Elektronen-Synchrotron DESY, Notkestraße 85, 22607 Hamburg, Germany \\ ${ }^{2}$ Department of Physics, Universität Hamburg, Luruper Chaussee 149, 22761 Hamburg, Germany \\ ${ }^{3}$ Center for Ultrafast Imaging, Universität Hamburg, Luruper Chaussee 149, 22761 Hamburg, Germany
}

\section{FRAGMENTATION CORRECTION OF MEASUREMENTS}

The strong-field-ionization technique employed in this work can lead to fragmentation, such that clusters from the molecular beam contributed to smaller masses in the mass spectrum (MS). For example, the water monomer and the water dimer signals at $m / q=18 \mathrm{u} / \mathrm{e}$ and $36 \mathrm{u} / \mathrm{e}$, respectively, contained contributions due to fragmentation of larger water-clusters in the molecular beam. Therefore, measured intensities needed to be corrected for these fragmentation channels. In addition, background water inside the chamber was measured at $18 \mathrm{u} / \mathrm{e}$ and needed to be corrected for.

For the latter, background measurement were permanently performed during the experiments using the higher repetition rate of the laser compared to the valve. Laser pulses were arriving in the interaction region at the same time as the molecular beam and between two molecular beam pulses, such that for each data point a background measurement was performed. The background signal was subtracted from the measurements.

The fragmentation ratios of the water dimer into smaller masses could be estimated and used for the calculation of the fraction of the water dimer in the deflected and undeflected molecular beam [1]. In Fig. 1 the deflection profiles measured at masses corresponding to $\mathrm{H}^{+}, \mathrm{O}^{+}$and $\mathrm{OH}^{+}$are shown. In the region of 2.8 to $3.5 \mathrm{~mm}$ the deflection curves look identical to those for the water dimer, indicating that at these positions those are fragments from the water dimer. The ratios of the water dimer to $\mathrm{H}^{+}, \mathrm{O}^{+}$and $\mathrm{OH}^{+}$at a position of $3 \mathrm{~mm}$ are $0.3,0.8$, and 0.7 , respectively. For the calculation of the fraction of the water dimer in the molecular beam for the undeflected beam, these ratios were used to estimate the amount of the water dimer inside of the beam.

For larger clusters, only fragments were measured, such that the measured signal was not solely due to a specific cluster stoichiometry and the overall shape of the molecular beam profile arose from several larger water-clusters. All protonated-water-cluster ions recorded showed the same deflection behavior, see Fig. 2. An estimate of the exponential decay of the measured protonated water-clusters distribution showed that protonated water-clusters $n=1-10$ contained $99.6 \%$ of the overall intensity.

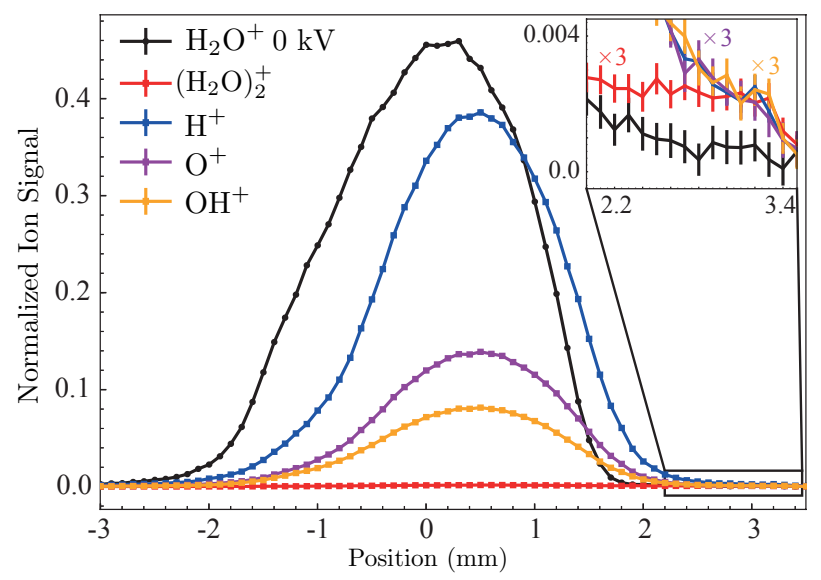

FIG. 1. Column density profiles, measured for the watermonomer cation with deflector voltages of $0 \mathrm{kV}$ (black) and for $\mathrm{H}^{+}$(blue), $\mathrm{O}^{+}$(purple), $\mathrm{OH}^{+}$(orange) and the water-dimer cation with a deflector voltage of $8 \mathrm{kV}$ (red). The inset shows the region around $y=3 \mathrm{~mm}$ enlarged, with $\mathrm{O}^{+}, \mathrm{OH}^{+}$and the water dimer ion scaled by a factor 3 .

\section{TRAJECTORY SIMULATIONS}

The Stark energies and effective dipole moments $\mu_{\text {eff }}$ of water-clusters $n=1 \ldots 7$ were calculated using the freely available CMIsTARK software package [2], which were then used to perform trajectory simulations [3] and to verify the measured deflection profiles of water-clusters. The rotational constants, dipole moments and centrifugal distortion constants from the literature are summarized in Table I. Three conformers for the water hexamer in prism-, book- and cage-like form [13] and two conformers of the water heptamer following the naming scheme of [15] were included.

For these simulations the water-clusters were assumed to be rigid rotors. Since the water dimer is known to be a floppy molecule with large amplitude motions [7, 16], the corresponding energy spectra and the description of the interaction of the states would significantly complicate further analysis. Using a rigid rotor assumption enables an easier and faster description and it has been shown previously that this model can be used to describe the dynamics of indole $\left(\mathrm{H}_{2} \mathrm{O}\right)$ in strong-electric- and laserfield alignment and orientation experiments [17, 18] and to fit pure rotational transitions of the water dimer to 


\begin{tabular}{|c|c|c|c|c|c|c|c|c|c|c|c|c|c|c|}
\hline \multirow[t]{2}{*}{ molecule } & \multicolumn{4}{|c|}{ dipole moment $\mu$ (D) } & \multicolumn{4}{|c|}{ rotational constants $(\mathrm{MHz})$} & \multicolumn{6}{|c|}{ Centrifugal Distortion constants (kHz) } \\
\hline & $\mu_{a}$ & $\mu_{b}$ & $\mu_{c}$ & & $A$ & $B$ & $C$ & & $\Delta_{J} \quad L$ & $\Delta_{J K} \quad \Delta$ & $\Delta_{K} \quad d$ & $d_{J} \quad d$ & $d_{K}$ & \\
\hline $\mathrm{H}_{2} \mathrm{O}$ & 0 & -1.86 & 0 & [4] & 835840.29 & 435351.72 & 278138.70 & [5] & $3.759 \times 10^{4}$ & $-1.729 \times 10^{5}$ & $9.733 \times 10^{5}$ & $1.521 \times 10^{4}$ & $4.105 \times 10^{4}$ & [5] \\
\hline$\left(\mathrm{H}_{2} \mathrm{O}\right)_{2}$ & 2.63 & 0 & 0 & [6] & 190327.0 & 6162.76 & 6133.74 & [7] & 0.044 & 4010 & 0 & 0 & 0 & [8] \\
\hline$\left(\mathrm{H}_{2} \mathrm{O}\right)_{3}$ & 0 & 0 & 0 & [9] & 6646.91 & 6646.91 & 0 & [10] & - & - & - & - & - & \\
\hline$\left(\mathrm{H}_{2} \mathrm{O}\right)_{4}$ & 0 & 0 & 0 & [9] & 3149.00 & 3149.00 & 1622.00 & [11] & - & - & - & - & - & \\
\hline$\left(\mathrm{H}_{2} \mathrm{O}\right)_{5}$ & 0.93 & 0 & 0 & [9] & 1859.00 & 1818.00 & 940.00 & [12] & - & - & - & - & - & \\
\hline$\left(\mathrm{H}_{2} \mathrm{O}\right)_{6}$ book & 0.17 & 2.46 & 0.16 & [13] & 1879.47 & 1063.98 & 775.06 & [13] & - & - & - & - & - & \\
\hline$\left(\mathrm{H}_{2} \mathrm{O}\right)_{6}$ cage & 1.63 & 0.32 & 1.13 & [13] & 2163.61 & 1131.2 & 1068.80 & [14] & - & - & - & - & - & \\
\hline$\left(\mathrm{H}_{2} \mathrm{O}\right)_{6}$ prism & 2.41 & 0.88 & 0.42 & [13] & 1658.22 & 1362.00 & 1313.12 & [13] & - & - & - & - & - & \\
\hline$\left(\mathrm{H}_{2} \mathrm{O}\right)_{7} 2$ & 1.0 & 0.0 & 1.0 & [15] & 1345.16 & 976.88 & 854.47 & [15] & 0.044 & 0.000 & 0.000 & 0.0000497 & 0 & [15] \\
\hline
\end{tabular}

TABLE I. Dipole moments, rotational constants and centrifugal distortion constants of water-clusters used in the Stark-effect calculations

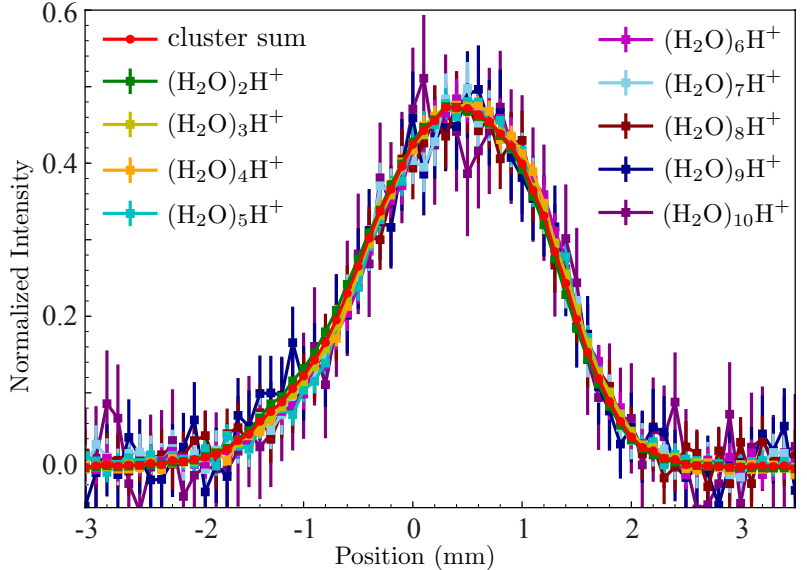

FIG. 2. Comparison of the averaged measured protonatedwater-cluster signal (red) and the individual measured protonated water-clusters deflection profiles for $n=2 \ldots 7$. The profiles are normalized to the area under the curve.

experimental measurements [8].

For the rotational states $J=0 \ldots 2$ of the water monomer and the water dimer the Stark energies and the corresponding $\mu_{\mathrm{eff}}$ as a function of the electric field strength are shown in Fig. 3. For the water dimer all relevant states are strong-field seeking and, hence, accelerated toward regions of stronger fields. For a nominal field strength of $50 \mathrm{kV} / \mathrm{cm}$ the $\mu_{\text {eff }}$ of the water dimer are significantly larger than for the water monomer, except from the $\left|J, K_{a}, K_{c}, M\right\rangle=|2,0,2,0\rangle,|2,1,1,1\rangle$ states, leading to a larger acceleration in the electric field. All the shown states have a small asymmetry splitting, see Table I, resulting in a fast rise of $\mu_{\mathrm{eff}}$ at small electric field strength. The discontinuous change of $\mu_{\text {eff }}$ at an electric field around $30 \mathrm{kV} / \mathrm{cm}$ is ascribed to an avoided crossing of the $|2,2,0,2\rangle$ and $|3,2,2,2\rangle$ states.

The trajectories of the molecules inside the electrostatic deflector were simulated using the calculated $\mu_{\text {eff }}[3]$. For quantum states $J=0 \ldots 10,10^{7}$ trajectories were calculated for each set of $J$ states and used to simulate the spatial profiles using a weighting factor based on the thermal distributions of the state for a given temper-

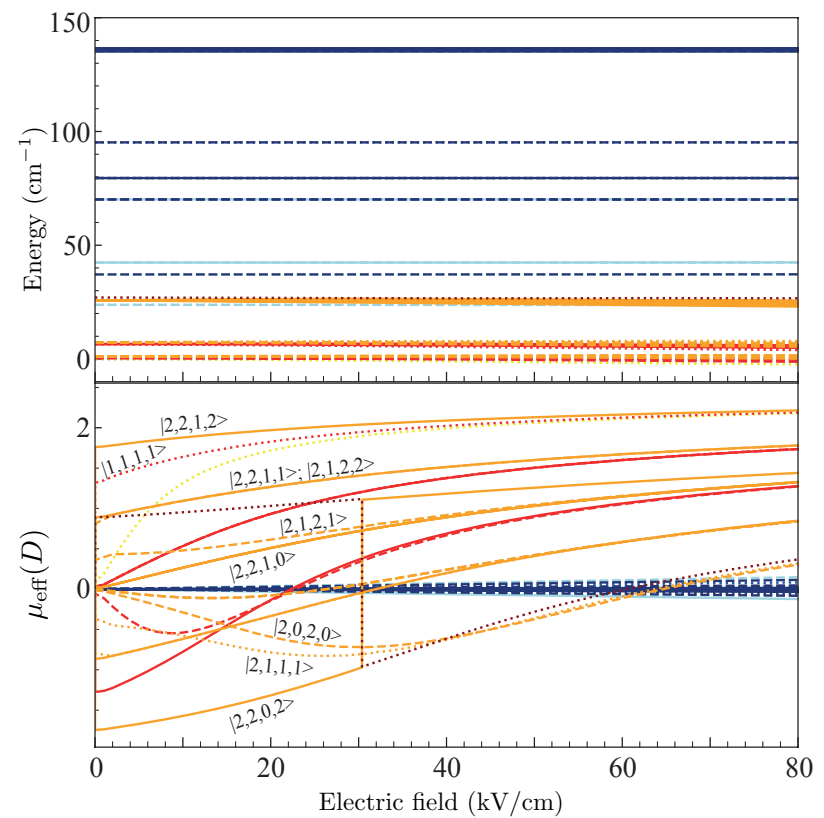

FIG. 3. Calculated Stark energy and effective dipole moments for the $J=0 \ldots 2$ states of the water monomer (blue) and the water dimer (red, orange, yellow). $J=0$ are shown in blue (water) and yellow (water dimer), $J=1$ in light blue (water), red (water dimer), $J=2$ in darkblue (water) and orange (water dimer) and the $|3,2,2,2\rangle$ state in darkred for the water dimer. States where $K_{a}<K_{c}$ are indicated by dashed lines, $K_{a}>K_{c}$ by solid lines and $K_{a}=K_{c}$ by dotted lines.

ature. Those temperature-weighted simulated vertical molecular-beam profiles were scaled to the area under the curve of the corresponding experimental profile to compare the deflection profiles. The simulations include the nuclear-spin-statistical weights for the water monomer and the water dimer. For para- and ortho-water a roomtemperature distribution of $1: 3$ was used. The water dimer in its equilibrium geometry is isomorphic with the permutation-inversion point group $D_{4 h}$ including tunneling splittings [19]. Neglecting tunneling splittings and acceptor switching, the rigid water dimer belongs to the symmetry group $C_{S}(M)$, yielding nuclear-spin-statistical 


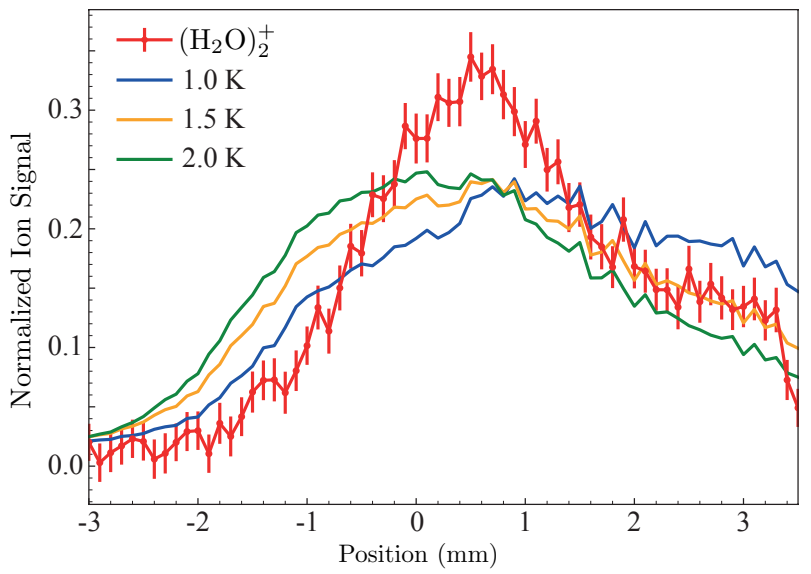

FIG. 4. Simulated deflection profiles for the water dimer at temperatures of $1.0 \mathrm{~K}, 1.5 \mathrm{~K}$ and $2 \mathrm{~K}$ compared to the corrected pure water dimer profile at $8 \mathrm{kV}$ (red, dots).

weights of para:ortho of $16: 16$ [20].

The simulated profiles for the water dimer at different rotational temperatures $T_{\text {rot }}$ including rotational states $J=0 \ldots 10$ are shown in Fig. 4. An initialbeam temperature of $T_{\text {rot }}=1.5(5) \mathrm{K}$ reproduced the experiment the best. At this temperature the water monomer in the para nuclear spin state has $100 \%$ of its population in its absolute rotational ground states $\left|J=0, K_{a}=0, K_{c}=0, M=0\right\rangle$, while ortho-water populates the $\left|J=1, K_{a}=0, K_{c}=1, M=0,1\right\rangle$ state to equal amounts. $99.9 \%$ of the para-water dimer and $99.9 \%$ of the ortho-water dimer population is within $J=0 \ldots 10$.

Trajectory simulations were performed for waterclusters up to $n=7$. Based on the estimated watercluster distribution, vide supra, this covers $97.8 \%$ of the water-clusters in the molecular beam, while $\sim 2.2 \%$ of the molecules in the beam are from water-clusters $n \geq 8$. The simulations for water-clusters including $J=0 \ldots 2$ and using the same rotational temperature $T_{\text {rot }}=1.5(5) \mathrm{K}$ of the water dimer are shown in Fig. 5. We note that at this temperature rotational states up to $J=10$ might be populated in the molecular beam and the rotational temperature can differ from the one of the water dimer. Thus the simulations give just an estimate of the amount of deflection. Based on the simulations the water dimer is deflecting the most of all water-clusters, followed by the water hexamer in prism- and book-like form, which reaches to a position of $+3.2 \mathrm{~mm}$.

Since for larger clusters only fragments have been measured and, therefore, the shape of the recorded beam profiles is the result of a superposition of several neutral cluster distributions in the molecular beam, it is not possible to compare the single deflection profiles directly with simulations. Therefore, at each position of the deflection profile the signal of the measured protonated water-clusters for $n=2 \ldots 10$ have been summed up. The

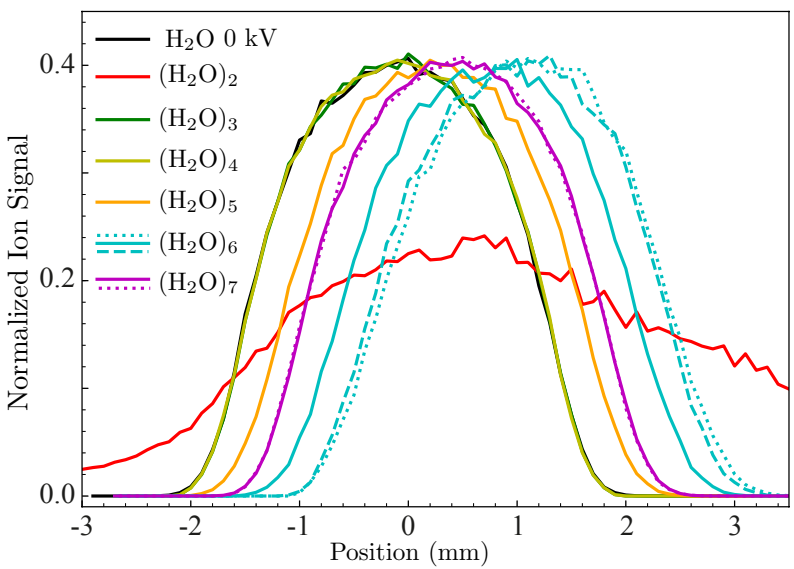

FIG. 5. Simulated deflection profiles for water-clusters for $n=2 \ldots 7$ are shown. Three different conformers of the water hexamer and two of the water heptamer were included, see text for details.

$\mathrm{H}_{3} \mathrm{O}^{+}, n=1$, contained also signal from the water dimer and has not been included. For the computationally derived profiles $n=3 \ldots 7$ were summed up for each position. As for the hexamer and heptamer several conformers have been simulated, each profile of the hexamer has been divided by 3 and for the heptamer by 2 . This is shown in Fig. 6. These simulations assume a rather low temperature of $1.5 \mathrm{~K}$ and did not include the needed nuclear spin statistical weighting for larger-clusters. In addition, the decaying water-cluster distribution in the molecular beam was not taken into account, resulting in a slightly different shape of the vertical molecular-beam-density profiles than

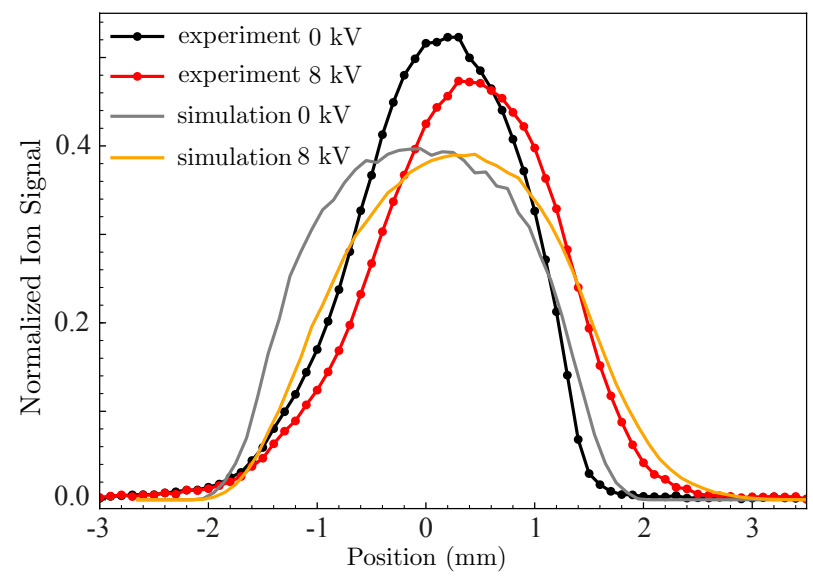

FIG. 6. Summed up simulated deflection profiles for waterclusters for $n=3 \ldots 7$ (grey, orange) and summed up measured deflection profiles for protonated water-clusters for $n=2 \ldots 10$ (black and red dots) for a deflector voltage of $0 \mathrm{kV}$ (grey, black) and $8 \mathrm{kV}$ (orange, red). The profiles are normalized to the area under the curve. 
the measured ones. However, comparing the simulated and the measured deflection profiles, the deflection is on the same order of magnitude and the right-hand side tail of the simulated deflection profile is reaching up to a position of $+3.2 \mathrm{~mm}$.

" Permanent address: Institute of Atomic and Molecular Physics, Jilin University, Changchun 130012, China

$\S$ Present address: Department of Chemistry, University of Basel, Klingelbergstrasse 80, 4056 Basel, Switzerland

$\ddagger$ Present address: Institute for Molecules and Materials, Radboud University, Heijendaalseweg 135, 6525 AJ Nijmegen, The Netherlands

* Corresponding author: jochen.kuepper@cfel.de; https://www.controlled-molecule-imaging.org

[1] M. Johny, J. Onvlee, T. Kierspel, H. Bieker, S. Trippel, and J. Küpper, "Spatial separation of pyrrole and pyrrolewater clusters," Chem. Phys. Lett. 721, 149-152 (2019), arXiv:1901.05267 [physics].

[2] Y.-P. Chang, F. Filsinger, B. Sartakov, and J. Küpper, "CMIstark: Python package for the stark-effect calculation and symmetry classification of linear, symmetric and asymmetric top wavefunctions in dc electric fields," Comp. Phys. Comm. 185, 339-349 (2014), arXiv:1308.4076 [physics].

[3] F. Filsinger, J. Küpper, G. Meijer, L. Holmegaard, J. H. Nielsen, I. Nevo, J. L. Hansen, and H. Stapelfeldt, "Quantum-state selection, alignment, and orientation of large molecules using static electric and laser fields," J. Chem. Phys. 131, 064309 (2009), arXiv:0903.5413 [physics].

[4] S. L. Shostak, W. L. Ebenstein, and J. S. Muenter, "The dipole moment of water. I. dipole moments and hyperfine properties of $\mathrm{H}_{2} \mathrm{O}$ and $\mathrm{HDO}$ in the ground and excited vibrational states," J. Chem. Phys. 94, 5875 (1991).

[5] F. C. DeLucia, P. Helminger, and W. H. Kirchhoff, "Microwave spectra of molecules of astrophysical interest V. Water vapor," J. Phys. Chem. Ref. Data 3, 211-219 (1974).

[6] N. P. Malomuzh, V. N. Makhlaichuk, and S. V. Khrapatyi, "Water dimer dipole moment," Russ. J. Phys. Chem. A 88, 1431-1435 (2014).

[7] L. H. Coudert and J. T. Hougen, "Analysis of the microwave and far infrared spectrum of the water dimer," J.
Mol. Spectrosc. 139, 259-277 (1990).

[8] T. R. Dyke, K. M. Mack, and J. S. Muenter, "The structure of water dimer from molecular beam electric resonance spectroscopy," J. Chem. Phys. 66, 498-510 (1977).

[9] J. K. Gregory, D. C. Clary, K. Liu, M. G. Brown, and R. J. Saykally, "The water dipole moment in water clusters," Science 275, 814-817 (1997).

[10] F. N. Keutsch, J. D. Cruzan, and R. J. Saykally, "The Water Trimer," Chemical Reviews 103, 2533-2578 (2003).

[11] J. D. Cruzan, L. B. Braly, K. Liu, M. G. Brown, J. G. Loeser, and R. J. Saykally, "Quantifying hydrogen bond cooperativity in water: Vrt spectroscopy of the water tetramer," Science 271, 59-62 (1996).

[12] K. Liu, M. G. Brown, J. D. Cruzan, and R. J. Saykally, "Vibration-rotation tunneling spectra of the water pentamer: Structure and dynamics," Science 271, 62-64 (1996).

[13] C. Pérez, M. T. Muckle, D. P. Zaleski, N. A. Seifert, B. Temelso, G. C. Shields, Z. Kisiel, and B. H. Pate, "Structures of cage, prism, and book isomers of water hexamer from broadband rotational spectroscopy." Science 336, 897-901 (2012).

[14] K. Liu, M. G. Brown, C. Carter, R. J. Saykally, J. K. Gregory, and D. C. Clary, "Characterization of a cage form of the water hexamer," Nature 381, 501-503 (1996).

[15] C. Pérez, S. Lobsiger, N. A. Seifert, D. P. Zaleski, B. Temelso, G. C. Shields, Z. Kisiel, and B. H. Pate, "Broadband fourier transform rotational spectroscopy for structure determination: The water heptamer," Chem. Phys. Lett. 571, 1 - 15 (2013).

[16] J. A. Odutola and T. R. Dyke, "Partially deuterated water dimers: Microwave spectra and structure," J. Chem. Phys. 72, 5062-5070 (1980).

[17] S. Trippel, Y.-P. Chang, S. Stern, T. Mullins, L. Holmegaard, and J. Küpper, "Spatial separation of state- and size-selected neutral clusters," Phys. Rev. A 86, 033202 (2012), arXiv:1208.4935 [physics].

[18] L. V. Thesing, A. Yachmenev, R. González-Férez, and J. Küpper, "Laser-induced alignment of weakly bound molecular aggregates," Phys. Rev. A 98, 053412 (2018), arXiv:1808.01206 [physics].

[19] T. R. Dyke, "Group theoretical classification of the tunneling-rotational energy levels of water dimer," J. Chem. Phys. 66, 492-497 (1977).

[20] P. R. Bunker and P. Jensen, Fundamentals of Molecular Symmetry, Series in Chemical Physics (Institute of Physics Publishing, Bristol, UK, 2005). 